\title{
Analysis of Software Engineering Database Programming Technology in Big Data Age
}

\author{
Pang Chonggao \\ School of Data Science and Computer Science, Guangdong Peizheng College, Guangzhou, Guangdong, \\ 510830, China \\ email: pangchonggao@163.com
}

Keywords: Big Data Era, Software Engineering Database, Programming Technology

\begin{abstract}
With the development of our country's current big data era, in software engineering database programming, relevant staff need to update and optimize the original software engineering database programming technology effectively according to the technology of big data, and construct the database programming model by using big data technology. Thus, the overall work can be well innovated. The related staff should constantly improve their technical level, understand the advanced software engineering database programming technology in the big data era, so as to effectively improve the efficiency of work.
\end{abstract}

\section{Analysis on Database Design and Construction of Based on Computer Software Engineering}

As an important part of the realization of computer office automation, computer software programming technology is also very important in the process of actual operation, which requires that the relevant staff should innovate the traditional programming mode effectively with more advanced technical means in the present era, use big data technology to improve the accuracy of software engineering database programming, and ensure the whole programming work is carried out in an orderly manner through the collection and processing of relevant data, thus reflecting the innovation of software engineering database programming technology in the current era. A database is a collection of data stored together in a certain way, shared by multiple users, with minimal redundancy and independent data from each other with the application. It contains integrity and sharing.

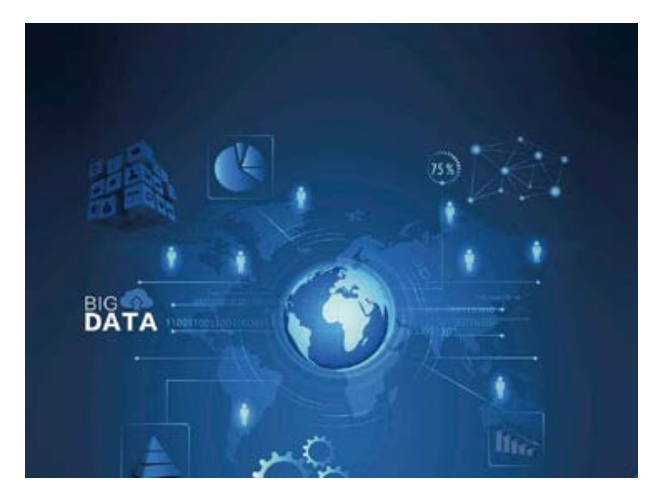

Figure 1 Software engineering database

\subsection{Database analysis based on computer software engineering}

In the process of applying computer software engineering database programming technology in the era of big data, the relevant staff should first make clear the components and important information of computer software engineering database, and in the process of actual design, the relevant data and confidence should be read comprehensively in the database file, and the relevant technicians should compare each other according to the important information and other information in the database, so as to select the best database information, combine the information 
in the database file and realize the best reading in the whole work, Comprehensive classification of the relevant data, so that the relevant risk data can be effectively controlled and other safety issues avoided [1]. After this series of work, we can participate in the construction of computer database through the unique way of reading information in the big data era.

In addition, in the design of computer database files, it is necessary for the relevant staff to compare the relevant data and other types of data in all directions, so that the next database file can be created. After the database file is created, the access rights and the type of information reading in the database have changed greatly, so it is necessary for the relevant staff to adopt the relevant design scheme according to the change of the computer database file in the actual work, so that the database can meet the actual needs and run normally in the computer software. At the same time, in order to avoid all kinds of problems in the process of database operation, relevant staff should make effective innovation and comprehensive construction of database system according to their ideas.

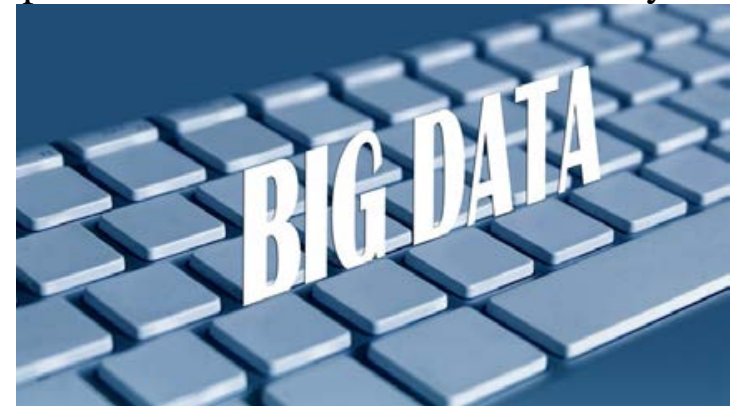

Figure 2 Database system

\subsection{Conceptual analysis of computer databases}

In order to ensure the effective application of the computer software engineering database programming technology, the relevant staff should make in-depth analysis and integration of the relevant concepts of the computer computer database in the process of practical work, and make use of the database file creation in the computer software engineering, which means that the relevant staff use the software programming technology of the computer software engineering to construct the database, thus forming a relatively complete database system. In the process of developing and utilizing the computer database, it is necessary for the relevant staff to make use of the advanced information technology in the current era of large data to make extensive use of the computer software. In order to meet the actual needs, comprehensive processing of relevant data and good storage, so as to optimize the development of computer database system [2]. In the process of computer database programming, we should fully understand the user's use requirements, the establishment of computer database for the user to carry out an automatic function of internal information processing system, can design and process the relevant data efficiently, so as to facilitate the user's use needs. In the computer database, the relevant staff need to provide users with a more efficient database processing platform to meet the diverse office needs of users and build the user range of the database.

Through the computer software, the whole database programming technology is optimized and maintained continuously, so that the relevant data information can be stored and transported efficiently to meet the needs of users. It can be seen that as a platform for information storage and exchange, the relevant staff must realize the efficient circulation and integration of relevant information, and combine the database with the computer office software, thus highlighting the role and value of the computer software engineering database programming technology in the big data era. 


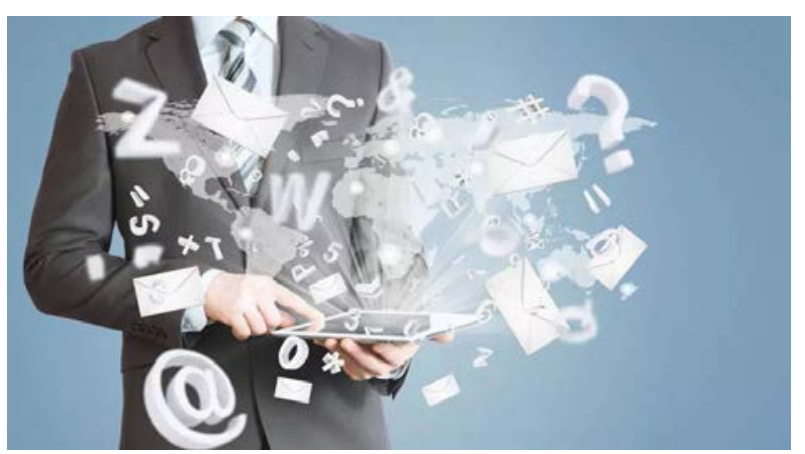

Figure 3 Computer software engineering

\section{Characteristics and Correction of Computer Software Engineering Database Programming Technology in the Big Data Age}

\subsection{Feature analysis of computer database software programming technology}

In the process of applying computer software engineering database programming technology in the era of big data, the relevant staff should first make clear the characteristics of computer database software programming technology, and in the process of designing and creating computer database, we must emphasize the application and maneuverability of database, so as to meet the actual needs of users to use database [3]. In the process of actual design and construction, the automatic repair speed of computer database should be accelerated, so that the carrying capacity of database can reach the expected standard. In the application of computer software engineering database programming technology, the related faults should be checked mainly from the data information interface, and the relevant staff should make a good backup in the process of actual work, and use the backup interface instead of the important information in the computer software engineering database, so as to help maintain the normal operation of the system. In the process of applying and supporting the computer software engineering database programming technology, if the network access of the database system is relatively large, then it is necessary for the relevant staff to screen the relevant important information at the backup interface, so as to ensure the normal operation of the database system, the backup interface in the database mainly undertakes part of the data processing and data transmission work, so that the balance of the carrying capacity of the whole database system can be realized, and the operation of the database system can be adjusted and controlled effectively in the course of the follow-up work.

At the same time, we should pay attention to the privacy and concealment of relevant data in the application of computer software engineering database programming technology. In the process of computer software running, the database mainly uses the network communication technology to realize the online communication, so the related staff can use the computer NAT technology to realize the secret processing of the data, and realize the whole network connection process through the public network URL in the process of database access. This design can not only satisfy the normal operation of the database in the state of network access, but also ensure the security of the database system effectively. In the process of computer software engineering database programming technology application, it is necessary to process the relevant data in privacy, most of the database in the process of practical application, most of the service for some enterprises, so in the process of database design, the internal and external network deployment can be used to carry out the actual construction. In this way, the enterprise can use the network to realize the exchange and communication of related resources, and the management of the enterprise can extract and understand some private data.

\subsection{Analysis of the Problems of Computer Software Engineering Database Programming Technology in the Big Data Age}

In the era of big data, there are still many problems in the computer software engineering database programming technology. In order to make the computer software engineering database 
programming technology more mature, the relevant staff should start with the problems of the computer software engineering database programming technology in the current big data era. The problem of database data security is becoming more and more prominent, which is because in the era of big data, the relevant staff need to realize the query and access of data in the process of database establishment using database programming technology. In the whole process of programming, the relevant data is easily modified or deleted, which leads to the high frequency and probability of occurrence of security problems in the whole process of programming, and there is a great threat to the normal operation of the database. Therefore, when using software engineering database programming technology, relevant technicians must break through this difficulty and improve the security of database establishment as a whole, so as to ensure that software engineering database programming technology can get stable development in the era of big data. As shown in the following figure, the relevant staff can improve the security of the database from the following nodes in the process of programming.

\section{Conclusion}

In the era of big data, relevant technicians must strengthen the research of computer software engineering database programming technology, fully understand the security problems of computer software engineering database technology in the era of big data, and make a comprehensive analysis of the application of programming technology in the application of programming technology, so as to ensure that computer software engineering programming technology can construct a relatively complete and secure database.

\section{References}

[1] Xing Junxin. Computer software development technology based on Java programming language. Electronic Technology and Software Engineering, no. 23, pp. 47-48, 2017.

[2] Wang Guigui. Database programming technology analysis based on computer software engineering. Software Applications, no. 21, pp. 3-4, 2018.

[3] Li sent ice. Analysis of the key technologies of software engineering in the era of big data. Academic Forum, no. 11, pp. 101-102, 2016. 